\title{
INCIDENCIA DE VIROSIS EN ÑAME (Dioscorea spp.) EN CINCO MUNICIPIOS DEL DEPARTAMENTO DE SUCRE, COLOMBIA
}

\author{
VIRUSES INCIDENCE ON YAM (Dioscorea spp.) IN FIVE \\ MUNICIPALITIES OF SUCRE, COLOMBIA INCIDENCIA DE \\ VIROSIS EN ÑAME
}

\author{
Iris R. Payares ${ }^{* 1}$, Javier D. Beltrán², Euriel Millán ${ }^{3}$ \\ Recibido para publicación: Enero 18 de 2014 - Aceptado para publicación: Mayo 9 de 2014
}

\begin{abstract}
RESUMEN
En Colombia, el ñame (Dioscorea spp.) se cultiva en la región Caribe, siendo Dioscorea alata L. y Dioscorea rotundata Poir. las especies más cultivadas. Uno de los factores limitantes en estos cultivos son las enfermedades causadas por virus, principalmente por Potyvirus que ocasionan disminución en la producción de los tubérculos y pérdidas económicas significativas. El objetivo de este trabajo fue evaluar la incidencia de virosis en ñame en el departamento de Sucre. Se realizaron visitas a cultivos de Dioscorea alata y Dioscorea rotundata en cinco municipios del departamento y se midió la sintomatología foliar. Se tomaron muestras de hojas y se realizó diagnóstico para Potyvirus mediante la técnica ELISA-DAS indirecta. Además, se inocularon plantas indicadoras con extractos preparados a partir de plantas con síntomas virales y positivas para Potyvirus. Todos los cultivos visitados mostraron síntomas asociados a los Potyvirus del ñame, tales como bandeo, moteado, clorosis y deformación foliar. Los municipios con mayor incidencia sintomática fueron Sampués $(82,5 \%)$, Sincelejo $(79,5 \%)$ y San Juan de Betulia $(71,6 \%)$. Se detectó Potyvirus en plantas sintomáticas y las plantas indicadoras desarrollaron síntomas similares a los encontrados en campo. Los cultivos de ñame del departamento de Sucre presentan alta incidencia de Potyvirus lo que sería causa de pérdidas económicas a los productores.
\end{abstract}

Palabras Clave: Virosis, Potyvirus, ELISA, Dioscorea alata, Dioscorea rotundata.

\begin{abstract}
In Colombia, yams (Dioscorea spp.) are grown in the Caribbean region, with Dioscorea alata L. and Dioscorea rotundata Poir. most cultivated species. One of the limiting factors in these crops are diseases caused by viruses, especially by Potyvirus that cause decreased production of tubers and significant economic losses. The aim of this study was to evaluate the incidence of yams viruses in the department of Sucre. Visits were made to crops Dioscorea alata and Dioscorea rotundata in five municipalities in the department and the presence of foliar symptoms was registered. Leaf samples were taken and Potyvirus diagnosis was performed by ELISADAS indirect technique. Furthermore, indicator plants were inoculated with extracts prepared from plants with viral symptoms and Potyvirus positive. All crops visited showed symptoms associated with Potyvirus yam, such as banding, mottling, chlorosis and leaf deformation. The municipalities with the highest symptomatic

\footnotetext{
'Bióloga con énfasis en Biotecnología.Laboratorio de Biología II.E-mail: iris.payares@unisucre.edu.co* Grupo de Investigación Evolución y Sistemática Tropical.

${ }^{2}$ PhD. Fitopatología. Docente Universidad de Sucre. E-mail: javier.beltran@unisucre.edu.co;Grupo de Investigación en Biotecnología Vegetal.

${ }^{3}$ MSc. Suelos y Nutrición Vegetal. Docente Universidad de Sucre. E-mail:euriel.millan@unisucre.edu.coGrupo de investigación recurso Suelo-Agua. Universidad de Sucre,carrera 28 No 5-267, Barrio Puerta Roja, Sincelejo, Colombia.
} 
incidence were Sampués (82.5\%), Sincelejo (79.5\%) and San Juan de Betulia (71.6\%). Potyvirus was detected in symptomatic plants and indicator plants, where developed symptoms similar to those found in the field. Crops yam of department of Sucre present high incidence of Potyvirus what would cause economic losses to producers.

Key words: Viruses, Potyvirus, ELISA, Dioscorea alata, Dioscorea rotundata.

\section{INTRODUCCIÓN}

El ñame pertenece a la familia Dioscoreaceae, género Dioscorea, desconociéndose con exactitud el número de especies. Varios autores, han reportado que existen entre 600 y 850 especies (Coursey 1967; AlShehbaz y Schubert, 1989, Perea y Buitrago 2000), pero gracias a estudios morfológicos y moleculares a nivel de género, se realizó una recircunscripción significativa del género Dioscorea reconociendo entre 350-400 especies (Caddick et al. 2002).

El cultivo de ñame es de importancia económica en regiones pluviosas tropicales y subtropicales, y sus tubérculos son considerados como producto esencial para la alimentación de millones de personas en África, Asia y América latina (Perea 2000; Osunde y Orhevba 2009) puesto que contienen importantes cantidades de carbohidratos y es fuente moderada de proteína y fibra (Blanco-Metzler et al. 2004; Alvis et al. 2008; Rugchati 2010). Colombia se encuentra dentro de los 12 países de mayor producción mundial, con 395.374 toneladas en 2010. Las áreas de cultivo se encuentran restringidas en su mayoría a la región Caribe con un 90\% de la producción nacional (Reina 2012), siendo Bolívar, Córdoba y Sucre los departamentos de mayor producción, donde es utilizado para consumo en fresco y exportación a los mercados de Estados Unidos y Europa (Sánchez y Hernández 1997; Alvarez 2000; Ministerio de Agricultura y Desarrollo Rural 2010).
Los cultivos de ñame pueden ser infectados por diferentes virus que generan pérdidas en la producción al disminuir el tamaño y la calidad de los tubérculos (Munford y Seal 1997). La disminución de la producción de los tubérculos de ñame ha sido poco evaluada. Thouvenel y Dumont (1990) estimaron la disminución de la producción de los tubérculos en $D$. alata cv. "Florido" en un $27 \%$ debido a la acción de el YMV (virus del mosaico del ñame).

En Guadalupe el YMV ha provocado perdidas en ñame entre un 10 y $30 \%$ y en Nigeria, los virus son responsable de pérdidas mayores a un 90\% (Malaurie et al. 1998). Según Amusa et al. (2003) el efecto de las enfermedades causadas por virus sobre el rendimiento de los tubérculos no se ha investigado suficientemente, pero sus observaciones plantean que bajo un severo ataque del virus de Dioscorea alata o enfermedad del virus del ñame de agua las perdidas pueden llegar a ser de más del 50\%.

Algunos géneros de virus reportados afectando ñame son: Potyvirus, Badnavirus, Cucomovirus, Comovirus, Potexvirus y Carlavirus (Kenyon et al. 2001; Asiedu et al. 2003; Seal y Muller 2007; Séka et al. 2009). Entre los Potyvirus se encuentran el Virus del mosaico del ñame (YMV) (Thouvenel y Fauquet 1979) y el Virus del mosaico suave del ñame (YMMV) (Munford y Seal 1997) que ha sido reportado como Virus de Dioscorea alata (DAV) (Odu et al. 1999), los cuales causan síntomas como mosaicos, moteados, bandeos y deformación foliar. El 
objetivo de este trabajo fue evaluar la incidencia de virosis a partir de la sintomatología en cultivos de ñame (Dioscorea alata y Dioscorea rotundata) de cinco municipios del departamento de Sucre, Colombia.

\section{MATERIALES Y MÉTODOS}

El estudio se realizó en los municipios de Corozal, Los Palmitos, Sampués, San Juan de Betulia y Sincelejo, en el departamento de Sucre y en el laboratorio de Fitopatología de la Universidad de Sucre. Estas localidades se eligieron por ser importantes áreas de cultivos de ñame del departamento (Umata 2009). Se realizaron muestreos en cultivos de las especies D. alata y D. rotundata, mediante recorridos en zig-zag, cuantificándose las plantas con sintomatología y sin sintomatología viral (Tabla 1), las que fueron marcadas para observar el desarrollo subsiguiente de los síntomas en el caso de las sintomáticas y la posible aparición de síntomas en las que no presentaban sintomatologías (Alvarez et al. 2011a), teniendo en cuenta los síntomas reportados para Potyvirus en ñame (Amusa et al. 2003; Eni et al. 2008). El porcentaje de incidencia se calculó mediante el cociente del número de plantas sintomáticas y el número de plantas muestreadas, multiplicado por cien (Chavarría 2004), adicionalmente, se calculó el porcentaje de plantas por sintomatología en cada localidad.

Se recolectaron muestras de hojas de la zona media de plantas sintomáticas de D. alata y D. rotundata y se analizaron 51 plantas mediante ELISA-DAS indirecto para Potyvirus, utilizando el kit comercial de Agdia. Se maceraron 0,5 g de material vegetal en $5 \mathrm{~mL}$ de buffer de extracción, se agregaron $100 \mu \mathrm{L}$ del extracto de cada planta a una placa de ELISA y se incubó por 12 horas a $4^{\circ}$ centígrados. Luego, se eliminó el extracto de la placa y se lavó tres veces cada pozo con PBS-T durante 5 minutos. Se adicionó $100 \mu \mathrm{L}$ de anticuerpo diluido 1:200 en buffer $\mathrm{ECl}$, se incubó durante 1 hora y se lavó tres veces con PBS-T. Se adicionó $100 \mu \mathrm{L}$ de anticuerpo conjugado en dilución 1:200 en buffer $\mathrm{ECl}$, se incubó durante 1 hora a temperatura ambiente y se lavó con PBS-T. Se adicionó la solución de PNPP, se incubó durante 1 hora en oscuridad y se realizó la lectura en un lector de ELISA (Daynatech MR 250) a $410 \mathrm{~nm}$. Cada muestra se analizó por duplicado y se usaron los controles negativos y positivos del kit. Una muestra fue considerada positiva cuando el valor de su absorbancia fue por lo menos dos veces el valor del control negativo.

Se realizó una prueba de trasmisión mecánica, sobre plantas indicadoras de Chenopodium amaranticolor, Nicotiana tabacum "sansum", Datura metel y Nicotiana rustica para corroborar lo encontrado por ELISA, siguiendo la metodología utilizada por Odu et al. (1999) y Hughes et al. (2000), para lo cual se utilizaron plantas que resultaron positivas para Potyvirus de D. alata mediante el ELISA-DAS indirecto. El jugo infectivo fue aplicado en 5 plantas indicadoras por especie y las plantas testigo fueron inoculados solo con buffer fosfato $0,1 \mathrm{M}$ con un arreglo factorial $2 \times 4 \times$ 5 (2 tratamientos, 4 especies y 5 repeticiones), para un total de 40 plantas. Para evaluar las variables especie y tratamientos $(\mathrm{T} 1=$ jugo infectivo y $\mathrm{T} 2$ = testigo) en el ensayo de transmisión mecánica se utilizó una Anova de dos factores mediante el programa G- stat 2.0 de licencia pública. 


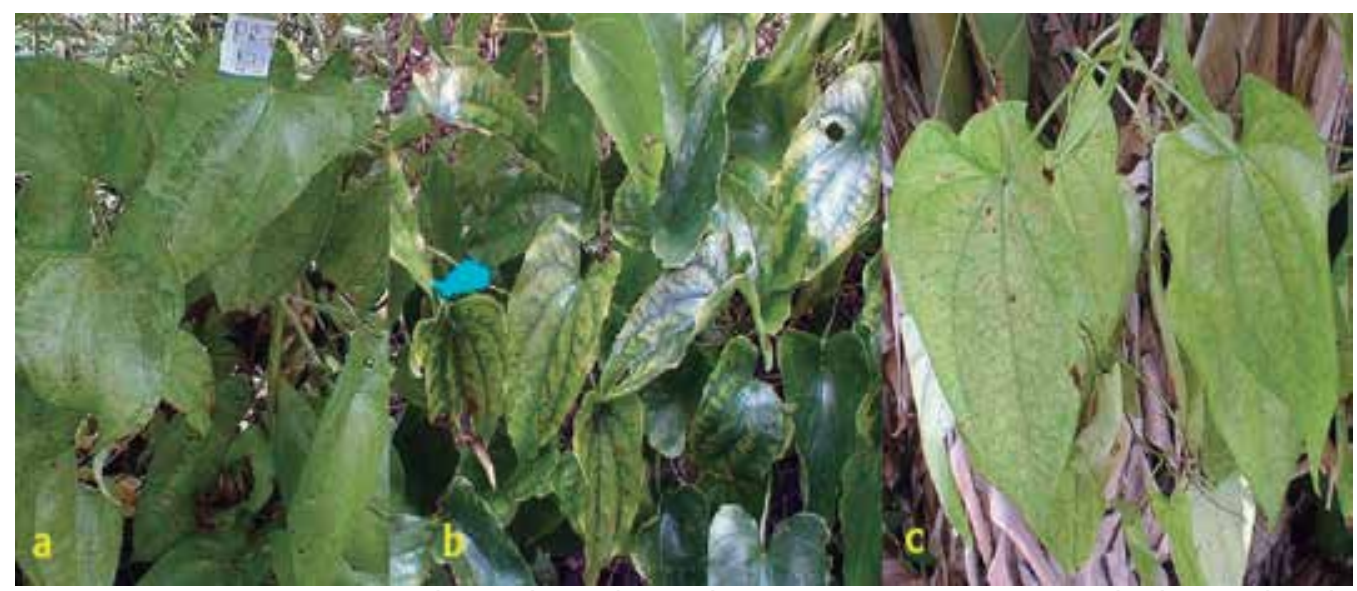

Figura 1. Síntomas encontrados en los cultivos de ñame en Sucre. a. Moteado; b. Bandeo de venas; c. Mosaico. Fuente: Autores.

Las observaciones sobre la aparición de sintomatologías se realizaron a partir del segundo día de haberse realizado la inoculación y la evolución subsiguiente de los síntomas se realizó a diario por siete semanas.

\section{RESULTADOS Y DISCUSIÓN}

Se observó sintomatología foliar de virosis en plantas de D. alata y D. rotundata de los cinco municipios visitados. D. alata presentó el mayor porcentaje de plantas con síntomas entre los municipios (Tabla1). Esto puede estar relacionado con la alta variabilidad genética de D. rotundata (Mignouna et al. 2002) que le proporciona mayor resistencia al ataque de patógenos que a D. alata. Pero también, debe tenerse en cuenta el sistema de producción, D. alata se cultiva asociado con Manihot esculenta Crantz, Zea mays L, Ipomoea batatas (L.) Lam, Cajanus cajan (L.) Huth y alguna cucurbitáceas, que se sabe son afectados por virus que también pueden atacar al ñame y convertirse en focos de infección adicionales (Brunt et al. 1996) mientras que D. rotundata en monocultivo lo que limita posibles focos de infección al no hallarse asociado a otras especies vegetales que pueden ser potenciales reservorios de éstos.

Los síntomas consistieron en moteado, bandeo de venas, mosaico, clorosis, retraso en el crecimiento y deformación foliar (Figura 1), y concuerdan con lo reportado para los Potyvirus del ñame (Goudou-Urbino et al. 1996; Amusa et al. 2003; Odu et al. 2004; Eni et al. 2008; Kajihara et al. 2008), y Badnavirus (Toualy et al. 2014).

Tabla1. Incidencia sintomática en cultivos de ñame del departamento de Sucre.

\begin{tabular}{ccccc}
\hline Municipio & Plantas muestreadas & $\begin{array}{c}\text { D. alata } \\
\text { Sintomáticas }\end{array}$ & $\begin{array}{c}\text { D. rotundata } \\
\text { Sintomáticas }\end{array}$ & $\begin{array}{c}\text { Incidencia } \\
(\mathbf{\%})\end{array}$ \\
\hline Sampués & 297 & 58 & 25 & 82,5 \\
Sincelejo & 420 & 41 & 38 & 79,5 \\
San Juan de Betulia & 250 & 52,8 & 18,8 & 71,6 \\
Corozal & 205 & 61,5 & 0 & 61,5 \\
Los Palmitos & 335 & 26 & 12 & 38 \\
\hline
\end{tabular}


Las mayores incidencias sintomáticas se presentaron en los municipios de Sampués y Sincelejo con un $82,5 \%$ y $79,5 \%$ respectivamente, seguidos de San Juan de Betulia (71,6\%), Corozal $(61,5 \%)$ y Los Palmitos (38\%), (Tabla 1). Los valores de incidencia medida a partir de la sintomatología asociada con virosis son altos y similares a los encontrados por Goudou-Urbino et al. (1996) en Burkina Faso en D. cayenensis-rotundata y D. alata, donde el porcentaje de incidencia en el área central del país fue de 71,5\%; por Oppong et al. (2007) quienes reportan hasta $80 \%$ de incidencia en el país de Ghana y por Toualy et al. (2014) en Costa de Marfil con una incidencia que varió entre 0 - 90\%.

Mediante el ELISA-DAS indirecto, seis (4 de D. alata y 2 de D. rotundata) de las 51 plantas analizadas resultaron positivas, lo que confirma la infección con Potyvirus de los cultivos de D. alata y D. rotundata en el departamento de Sucre, y 45 fueron negativas, pero de ésas, 43 pese a estar por debajo del punto de corte presentaron valores superiores al control negativo, esto hace considerar que es posible una coinfección con otros agentes patógenos, incluyendo diferentes géneros de virus que se sabe infectan al ñame, por lo que es necesario aplicar pruebas diagnósticas más específicas y con mayor sensibilidad como ICRT-PCR e ISEM (Njukeng et al. 2005; Kajihara et al. 2009).

En el ensayo de transmisión mecánica en plantas indicadoras, la aparición de los síntomas ocurrió 14 días después de iniciado el ensayo, presentándose aclaramiento de nervaduras y bandeo intervenal en plantas de N. rustica, mosaico suave en N. tabacum "sansum"

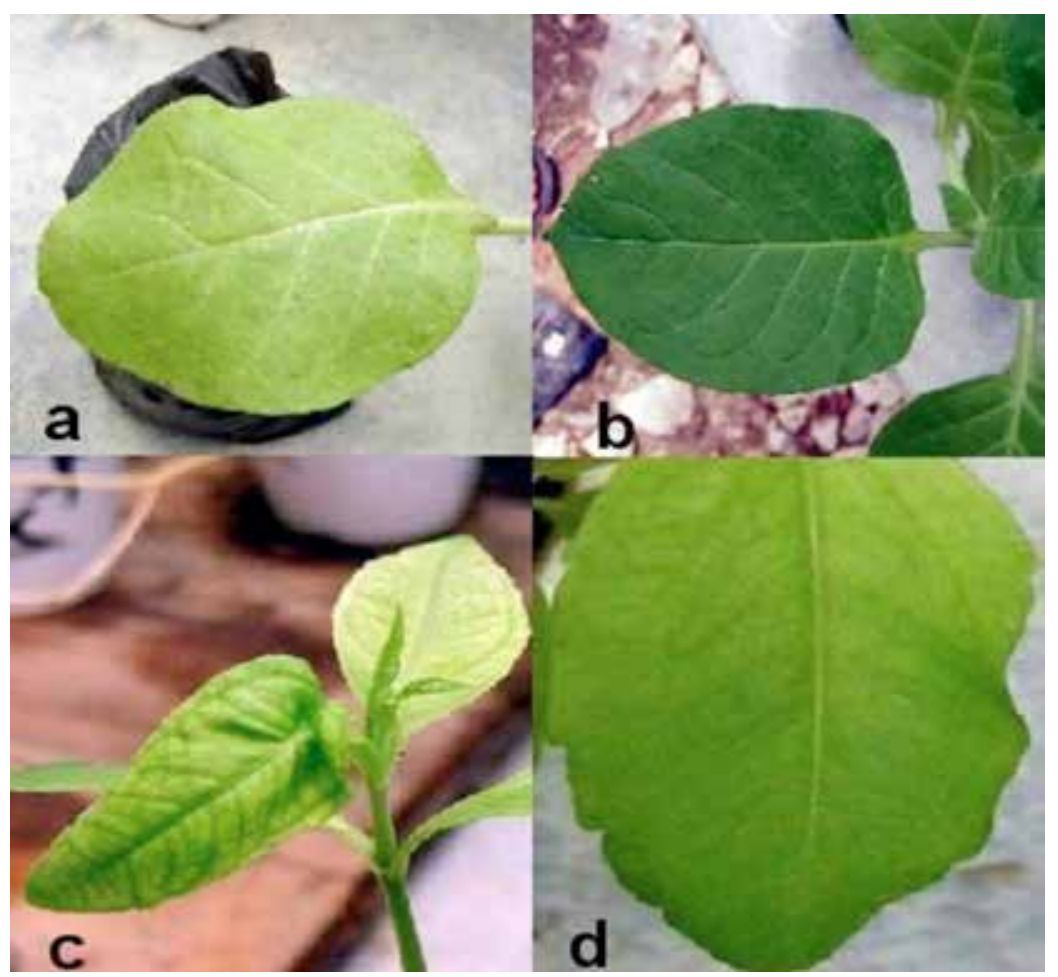

Figura 2. Síntomas en plantas indicadoras: a. Mosaico suave en N. tabacum "Samsum", b. Testigo, c. Bandeo, clorosis y enroscamiento en Chenopodium amaranticolor, d. Testigo. Fuente: Autores. 
(Véase figura 2a y 2b); bandeo intervenal con diferentes grados de clorosis y aclaramiento de nervaduras en Ch. amaranticolor que después se hizo más conspicuo (Figura 2c y 2d).

La evaluación final del ensayo se realizó 50 días después del inicio de éste. Las plantas de N. tabacum "sansum", N. rustica inoculadas con el jugo infectivo mostraron síntomas en un $100 \%$, y Ch. amaranticolor en un 80\% (Tabla 2).

Al finalizar el ensayo, se pudo observar necrosis de hojas intermedias y deformación de las hojas apicales. Las plantas testigo no presentaron ningún tipo de sintomatología. D. metel no presentó síntomas (Tabla 2). Se ha documentado que ésta especie puede ser susceptible o no susceptible dependiendo de la cepas de Potyvirus, Sobemovirus, Comovirus, Tobamovirus o Carlavirus que entren en contacto con ella (Rosas 2004; Peña y Trujillo 2007; Al-Khatib 2008; Blotskaya y Vabishchevich 2011).

El análisis de varianza mostró que existe diferencia altamente significativa entre las plantas tratadas con el jugo infectivo $y$ las testigos $\mathrm{p}<0.0007 \mathrm{E}-4$. Las pruebas de transmisión mecánica mediante el uso de plantas indicadoras, se realizó para explorar la posibilidad de usar esta técnica como apoyo en nuestro medio para detectar virus en ñame y en otras especies económicamente importantes al no ser costoso, relativamente fácil de llevar a cabo y presentar resultados en corto tiempo, lo que puede permitir realizar controles fitosanitarios rápidos sin tener que contar con demasiados recursos y poder tomar decisiones de manejo del cultivo adecuadamente.

Investigaciones como las realizadas por Dallot et al. (2001), Guzmán et al. (2001), Alvarez et al. (2011a) y Alvarez et al. (2011b) sugieren una alta incidencia de Potyvirus en los cultivos de ñame en la región Caribe de Colombia, y reportan síntomas como moteado, mosaico, bandeo de venas, corrugamiento y deformación foliar; así como la infección de estos con el Virus del mosaico suave del ñame. Se ha demostrado que los Potyvirus del ñame pueden ser transmitidos mediante tubérculos infectados utilizados como material de siembra (Thouvenel Fauquet 1979; Goudou-Urbino et al. 1996; Guzmán et al. 2001; Odu et al. 2004), esta situación sumada a la presencia de cicadélidos vectores (Alvarez et al. 2011a;

Tabla 2. Porcentaje de infección en plantas indicadoras infectadas con jugo infectivo de plantas de D. alata positivas para Potyvirus.

\begin{tabular}{lcc}
\hline \multicolumn{1}{c}{ Especie } & \% de infección & IPS/PI \\
\hline N. tabacum var. samsum & 100 & $5 / 5$ \\
N. tabacum var. samsum Testigo & 0 & $0 / 5$ \\
Ch. Amaranticolor & 80 & $4 / 5$ \\
Ch. amaranticolorTestigo & 0 & $0 / 5$ \\
N. rustica & 100 & $5 / 5$ \\
N. rústicaTestigo & 0 & $0 / 5$ \\
D. metel & 0 & $0 / 5$ \\
D. metel & 0 & $0 / 5$
\end{tabular}

PS = plantas con síntomas; PI = plantas inoculadas. 
Alvarez et al. 2011b) y de arvenses hospederos alternativos de virus, podría ser la causa de la alta incidencia de síntomas asociados con virosis encontrado en los cultivos de ñame en el departamento de Sucre.

\section{CONCLUSIONES}

Los cultivos de ñame del departamento de Sucre presentaron una alta incidencia de plantas con síntomas asociados a Potyvirus, aunque mediante el ELISA-DAS indirecto solo seis plantas resultaron positivas y 45 fueron consideradas negativas, pero de éstas 43, presentaron valores superiores a el control negativo pero sin alcanzar el valor del control positivo, lo que deja ver la necesidad de realizar pruebas más sensibles pues existe la posibilidad que otros géneros de virus se encuentren presentes en los cultivos de ñame del departamento de Sucre. El hecho que se hayan encontrado plantas positivas para Potyvirus hace considerar que las enfermedades causadas por virus pueden estar afectando la producción y causando pérdidas económicas a los productores aún sin cuantificar.

\section{REFERENCIAS}

Al-Khatib, H. 2008. Use of Programmed Cell Death Symptom and Physiological Changes for Tomato Cultivars as MarkerAssisted Selection for Plant Acquired Resistance to Tomato Infected With Tomato Mosaic Tobamovirus. Tesis Master of Science (M. Sc.)/ (Microbiology /Plant Virology). King Saud University. College of Science. Department of Botany and Microbiology.
Al-Shehbaz, I. and Schubert, B. 1989. The Dioscoreaceae in the Southeastern United States. Journal of the Arnold Arboretum . 70: 57-95.

Alvarez, D., Pérez, A., Díaz, J., Maestre, M. y Beltrán, J. 2011a. Transmisión del Virus del mosaico suave del ñame a Dioscorea rotundata (Dioscoreaceae) por Oncometopia sp. (Cicadellidae). Rev. Colomb. Entomol. 37: 77-79.

\section{Alvarez, D., Pérez, A., Díaz, J., y Beltrán, J.} 2011b. Detección del Virus del mosaico suave del ñame mediante IC-RT-PCR en cicadélidos, Rhynchosia minima $y$ Dioscorea rotundata. Temas Agrarios. 16: 36-40.

Alvarez, A. 2000. Prácticas agronómicas para el cultivo del ñame. En: Guzmán, M. \& Buitrago, G. (eds.). Ñame: producción de semilla por biotecnología. Unibiblos. Bogotá, Colombia. p33-39.

Alvis,A.,Vélez, C.y Rada, M.2008. Composición de ñames frescos cultivados en Colombia y sometidos a freído por inmersión. Inf. Tecnol. 19: 3-10.

Amusa, N., Adegbite, A., Muhammed, S. y Baiyewu, R. 2003. Yam disease and its management in Nigeria.Afr. J. Biotecnol.2(12): 497-502.

Asiedu, R., Mignouna, H., Odu, B. y Hughes, J. 2003. Yam breeding. En: Plant virology in sub-Saharan Africa. International Institute of Tropical Agriculture (IITA), Ibadan, Nigeria. p466-475. 
Blanco, A., Tovar, J. and Fernández, M. 2004. Caracterización nutricional de los carbohidratos y composición centesimal de raíces y tubérculos tropicales cocidos, cultivados en Costa Rica. ALAN 54 (3): 322-327.

Blotskaya, Zh. y Vabishchevich, V. 2011. Viral diseases of tomatoes and cucumbers in greenhouses of Belarus.БІологія. 59. p4-6.

Brunt, A., Crabtree, K., Dallwitz, M., Watson, L. and Zurcher, E. (rds). (1996). Plant Viruses Online: Descriptions and lists From the Vide Database. Dioscorea green banding mosaic Potyvirus. p1-4.

Caddick, R., Wilkin, P., Rudall, P., Henderson, T. and Chase, M. 2002. Yams reclassified: a recircumscription of Dioscoreaceae and Dioscoreales. Taxon 51: 103-114.

Chavarría, M. 2004. Evaluación de cinco variedades de tomate (Lycopersicum esculentum Mill) en relación al complejo mosca blanca- Geminivirus bajo infecciones naturales en la zona del pacifico de Nicaragua. Tesis de Ing. Agrónomo. Managua, Nicaragua. UNA FAGRO. p3-4.

Coursey, D. 1967. Yams. Dioscorea spp (Dioscoreaceae). En: Simmons NW. (Ed) Evolution of crop plants. Longman, London. p70-74.

Dallot, S., Bousalem, M. y Guzman, M. 2001. Occurrence of potyviruses on yam (Dioscorea spp) in Colombia and first molecular characterization of Yam mild mosaic virus. Plant Dis. 85(7): 803 p.
Eni, A., Hughes, J. and Rey, M. 2008. Survey of the incidence and distribution of five viruses infecting yams in the major yamproducing zones in Benin. Ann. Appl. Biol. 153: 223-232.

Goudou, C., Konate, G., Quiot, J. and Dubern, J. 1996. Aetiology and ecology of a yam mosaic disease in Burkina Faso. Trop. Sct. 36: 34-40.

Guzmán, M., Bermudez, Y. y Castro, C. 2001. Identificación del Virus del mosaico suave del ñame(YMMV) en muestras colombianas de Dioscorea alata caracterización biológica, serológica y molecular. Rev. Colomb. Biotecnol. 3: 72-79.

Hughes, Jd'A., Meerman, JC., Speijer, PR., Vernier, P., Asiedu, R., en colaboración con Dongo, L., Kwoseh, C., Mudiope, J., Olatunde, O., Tchabi, A., Atiri, G., Kenyon, L., Peters, J., Offei, SK., Plowright, R. and Gumedzoe, M. 2000. Evaluate distribution and severity of yam pests and diseases in West Africa. Viruses. Project 13. Improvement of yam-based production systems. IITA.

Kajihara, H., Muramoto, K., Fuji, S., Tanaka, S. and Ito, S. 2009. Simultaneous detection of Japanese yam mosaic virus and yam mild mosaic virus from yam leaves using a tube capture reverse transcriptionpolymerase chain reaction assay. J. Gen. Plant. Pathol. 75: 72-75.

Kajihara, H., Kameya, M., Oonaga, M., Kimura, I., Sumida, Y., Ooi, Y. and Ito, S. 2008. Field studies on Cross-protection 
against Japanese yam mosaic virus in Chinese yam (Dioscorea opposita) with an Attenuated Strain of the Virus. J. Phytopathol. 156: 75-78.

Kenyon, L.; Shoyinka, S., Hughes, J.d'A. and Odu, B. 2001. An overview of viruses infecting Dioscorea yams in sub-Saharan Africa. En: Hughes, J.d'A. and Odu,B.O. (Eds.). Plant Virology in Sub-Saharan Africa. Proceeding of a conference organized by IITA. p432-439.

Malaurie, B, Trouslot, M., Berthaud, J., Bousalem, M., Pinel, A and Dubern, J. 1998. Mediumterm and long-term in vitro conservation and safe international exchange of yam (Dioscorea spp) germplasm. Electronic Journal of Biotechnology. Vol.1 No 3.

Ministerio de Agricultura y Desarrollo Rural. 2010. Anuario estadístico del sector Agropecuario y pesquero. Dirección de política sectorial- Grupo sistemas de información. Bogotá D.C. Colombia. 262p.

Mignouna, H., Dansi, A. and Zok, S. 2002. Morpho-logical and isozymic diversity of the cultivated yams (Dioscorea cayenensis/Dioscorea rotundata complex) of Cameroon. Genet. Resource. Crop Evol. 49: 21-29.

Munford, R. and Seal, S. 1997. Rapid single tube immunocapture RT-PCR for the detection of two yam potyviruses J. Virol. Methods. 69: 73-79.

Njukeng, A., Atiri, G. and Hughes, J. 2005. Comparison of TAS-ELISA, Dot and Tissue
Blot, ISEM and inmunocapture RT-PCR assays for the detection of Yam Mosaic Virus in yam tissues. Crop Protection. 24: 513-519.

Odu, B., Hughes, J., Asiedu, R., Shoyinka, S. and Oladiran, O. 2004. Responses of white yam (Dioscorea rotundata) cultivars to inoculation with three viruses. Plant Pathol. 53: 141-147.

Odu, B., Hughes, J., Shoyinka, S. and Dongo, L. 1999. Isolation, characterization and identification of Potyvirus from Dioscorea alata L. (water yam) in Nigeria. Ann. Appl. Biol. 134: 65-71.

Oppong, A., Lamptey, J., Ofori, F., Anno, F., Offei, S. and Dzomeku, B. 2007. Serological detection of Dioscorea alata Potyvirus on whie yams Dioscorea rotundata in Ghana. J. Plant. Sci. 2(6): 630-634.

Osunde, Z. and Orhevba, B. 2009. Effects of storage conditions and storage period on nutritional and other qualities of stored yam (Dioscorea spp) tubers. Afr. J. Food Agric. Nutr. Dev. 9(2): 678-690.

Perea, M. y Buitrago, G. 2000. Aplicación de la biotecnología agrícola al cultivo de ñame. pp. 17-32. En: Guzmán, M. \& Buitrago, G. Ñame: producción de semilla por biotecnología. Unibiblos. Bogota. Colombia.

Perea, M. 2000. Utilización de los sistemas in vitro para la obtención de plantas de ñame (Dioscorea spp) libres de 
patógenos. En: Guzmán, M. \& Buitrago, G. Name: producción de semilla por biotecnología. Unibiblos. Bogota. Colombia. p41-53.

Peña, Z. y Trujillo, G. 2007. Identificación de virus que se transmiten a través de semillas de caraota (Phaseolus vulgaris (L.) y fríjol (Vigna unguiculata (L.) Walpers) en áreas productoras de Venezuela. Rev. Fac. Agron. (LUZ). 24: 1-21.

Reina, Y. 2012. El cultivo de ñame en el Caribe colombiano. Documentos de Trabajo Sobre Economía Regional. No. 168. Banco de la República. 34 p.

Rosas, T. 2004. Estudio comparativo de especies del género Carlavirus que afectan al cultivo de la papa. Tesis Bióloga con mención en Microbiología y Parasitología. Universidad Nacional Mayor de San Marco. Facultad de ciencias Biológicas.

Rugchati, O. 2010. Comparasion in some characteristic of yam tubers starch (Dioscoraceae spp.) from Thailand. International Journal of environmental and Rural development. 1-2. p102-106

Sánchez, C. y Hernández, L. 1997. Descripción de aspectos productivos, de postcosecha y de comercialización del ñame en Córdoba, Bolívar y Sucre. Temas Agrarios. 2(4): 105-120.
Seal, S. and Muller, E. 2007. Molecular analysis of a full-length sequence of a new yam badnavirus from Dioscorea sansibarensis. Arch. Virol. 152: 819-825.

Séka, K., Diallo, A., Kouassi, K. and Aké, S. 2009. Incidence du Yam Mosaic Virus (YMV) et du Cumcuber Mosaic Virus (CMV) sur des variétés de Dioscoreas spp. cultivées dans les régions de Bouaké et de Toumodi en Côte d'Ivoire. Inter. J. Biologic. Chemic. Sci. 3: 694-703.

Thouvenel, J. y Dumont, R. 1990. Pertes de rendement de L`igname infectée par le virus de la mosaïque de ligname en Côte d’Ivoire. Agro. Trop. 45. 125-129.

Thouvenel, J. and Fauquet, C. 1979. Yam mosaic Potyvirus infecting Dioscorea cayenensis in the Ivory coast. Ann. Appl. Biol. 93: 279-283.

Toualy, M., Diallo, H., Akinbade, S., Séka, K. and Kumar, P. 2014. Distribution, incidence and severity of viral diseases of yam (Dioscorea spp.) in Côte d'Ivoire. African Journal of Biotechnology. 13(3): 465-470.

\section{Unidad municipal de asistencia técnica agropecuaria (Umata). 2009. Corozal, Los Palmitos, Sampués, San Juan de Betulia y Sincelejo.}

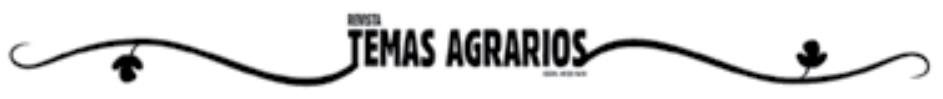

